\title{
Dynamics of Small Spin Polaron in the Three-Band Model of Two-Dimensional Spherically Symmetric Antiferromagnet.
}

\author{
A.F. Barabanov \\ Institute for High Pressure Physics, \\ Troitsk, Moscow region, 142092, Russia. \\ R.O. Kuzian \\ Institute for Materials Science, \\ Krjijanovskogo 3, Kiev, 252180, Ukraine \\ L.A. Maksimov \\ Russian Research Center, Kurchatov Institute, \\ Kurchatov sq.46, Moscow, 123182, Russia. \\ E. Z̆a̧sinas \\ Institute for High Pressure Physics, \\ Troitsk, Moscow region, 142092, Russia.
}

()

\begin{abstract}
The retarded Green's function $G(\mathbf{k}, \omega)$ of a single small spin polaron in the three-band model for the $\mathrm{CuO}_{2}$ plane is calculated in the self-consistent Born approximation. It is shown that such a spin polaron is a good quasiparticle excitation for realistic values of spin exchange $J$ and effective hopping $\tau$. The polaron spectral density $A_{p}(\mathbf{k}, \omega)$ demonstrates small damping in contrast to the results of calculations starting from the bare hole, i.e. the pole strength of the energetically low-lying quasiparticle peak $Z_{p}(\mathbf{k})$ varies from $50 \%$ to $82 \%$
\end{abstract}


for $J / \tau \propto 0.1 \div 0.7$. The quasiparticle peak dispersion reproduces the main features of the bare polaron spectrum $\Omega_{k}$ near the band bottom.

The spherically symmetric approach is used for the description of spin excitations. It makes it possible to consider the quantum antiferromagnetic background without the spontaneous symmetry breaking and the unit cell doubling.

The new method of the self-consistent calculation, based on continued fraction expansion of Green's function, is represented in details. The method preserves the proper analytical properties of the Green's function and provides the possibility to analyze the nature of its singularities.

71.27.+a,71.10.Fd,75.30.Mb

Typeset using REVTEX 


\section{INTRODUCTION}

Up to date much theoretical work has been devoted to the problem of a hole motion in two dimensional (2D) $\mathrm{s}=1 / 2$ quantum antiferromagnet (AFM) [1]. The important question is whether a hole injected in the undoped ground state behaves like a quasiparticle (QP). This problem is mainly investigated within the framework of self-consistent Born approximation (SCBA) for the $t-J$ model [2] [7] and Kondo-lattice [8]. There are rather few works devoted to the three-band Hubbard model or the Emery model [9, 10] that is more realistic for $\mathrm{CuO}_{2}$ planes in high- $T_{c}$ superconductors (HTSC). For the $t-J$ model it was shown that the spectral density function $A_{h}(\mathbf{k}, \omega)$ of a doped hole revealed a QP peak of intensity $Z_{\mathbf{k}} \approx J / t$ and a broad incoherent part that has a width of about $6-7 t$. The QP band bottom corresponds to the momenta $\mathbf{k}_{1}=( \pm \pi / 2, \pm \pi / 2)$. Similar results were obtained for the Emery model [11,12]. The presence of a great incoherent part and small intensity of QP peak indicate, that bare holes are rather poor elementary excitations even for $\mathbf{k}$ close to $\mathbf{k}_{1}$

In order to investigate the hole motion in the $t-J$ model one usually decouple the hole operator into a spinless fermion and an antiferromagnetic magnon operator. As a result the zero approximation corresponds to the dispersionless band with zero energy of the hole. The hopping of the particle appears only due to the fermion-magnon scattering which is treated by the usual perturbation method in $\mathbf{k}$-space. For this reason we think that in this approach the resulting quasiparticle pole in the fermion Green's function takes into account mainly a polaron of a large radius. The close situation takes place in the usual treatment of a hole

motion in the effective three-band model [11 [13] and the Kondo-lattice model [8], when one starts from a bare hole, not from a magnetic polaron of a small radius.

In the present paper, within the framework of the effective three-band model we study the spectral density function $A_{p}(\mathbf{k}, \omega)$ of a single small polaron,i.e., an excitation which takes into account from the very beginning a local hole-spin coupling . It is well known that the simplest candidate for such a small polaron is an analog of the so-called Zhang-Rice singlet in $\mathrm{CuO}_{4}$ 
plaquette [14], [15]. The mean-field spectrum $\Omega_{\mathbf{k}}$ of this excitation is well studied [15] and it will be used as the zero approximation in our treatment. We shall consider the coupling of small polaron to spin-wave excitations within the self-consistent Born approximation (SCBA) for the corresponding two-time retarded Green's function $G(\mathbf{k}, \omega)$.

Our motivations to study $A_{p}(\mathbf{k}, \omega)$ and the corresponding quasiparticle band are the following. First, it may be easily shown for the one-hole problem that the mean-field energy of the polaron $\Omega_{\mathbf{k}}$ represents the center of gravity of the spectral function

$$
\Omega_{k}=\int_{-\infty}^{\infty} \omega A_{p}(k, \omega) d \omega
$$

This means that the minimum of $\Omega_{\mathbf{k}}$ is the upper bound of the actual position for the quasiparticle band bottom. The SCBA, based on a bare hole Green's function, gives the minimum value of quasiparticle energy equal to $\omega_{h, \min }=-2.6 \tau$ [12], for a typical value of copper-copper AFM exchange constant $J=0.7 \tau$. Here $\tau$ is a constant of the effective oxygen-oxygen hopping via an intervening copper site (note that our unit of energy is twice that of Ref. [12 $\tau=2 t$ ). As to the value of the small polaron mean field band bottom it turns out to be substantially lower than $\omega_{h, \min }, \Omega_{\mathbf{k}}=-3.17 \tau$, for the same value of $J / \tau$. So we may conclude that important local correlations are lost in SCBA when we start from the bare hole operators.

Secondly, we shall show that a small polaron represents the elementary hole excitation much more better than a bare hole dressed by magnons within the framework of SCBA. This will be manifested by a relatively great intensity of a quasiparticle peak in our calculation.

Finally, the mean field spectrum $\Omega_{\mathbf{k}}$ of the simplest small spin polaron explicitly depends on the state of the antiferromagnetic background. In the case of long range order state, $\Omega_{\mathbf{k}}$ demonstrates a flat band region close to the magnetic Brillouin zone boundary [15]. This region corresponds to the bottom of the band. Moreover, if one takes into account the direct oxygen-oxygen hopping, finite temperature and a more complicated form for a small polaron wave function, then $\Omega_{\mathbf{k}}$ reproduces the experimentally observed extended saddle point [16 20] which is directed along the line $(0, \pi)-(0,0)$ [21]. Therefore it seems 
important to clear up if the quasiparticle band reproduces the peculiarities of $\Omega_{\mathbf{k}}$ dispersion. Below we study this question for the simplest variant of the model.

The distinctive feature of our investigation consists in considering the AFM copper spin subsystem in a spherically symmetric approach [22,23]. Such an approach is the most appropriate for treating the quantum 2D AFM at any finite temperature. As a result the scattering of a spin polaron by spin excitations in the singlet spin background leads to the spectral function periodicity relative to the full Brillouin zone. Note that the conventional two-sublattice spin approach leads to periodicity relative to the magnetic (reduced) Brillouin zone [2, 0,12$]$.

The paper is organized as follows. In Section II we give the derivations for the selfconsistent equation for the Green's function in the case of the small polaron approach. In Section III we present the procedure that provides the opportunity to avoid the iterative solution of the self-consistent equation for complex energies. The procedure is based on the continued fraction (CF) expansion of Green's function and it gives a possibility to calculate consequently the coefficients of the CF expansion with the use of the quadrature method. In Section IV we offer the termination of the continued fraction which leads to the correct analytical properties of the resulting Green's function. Numerical results for the self-energies and spectral functions, the relation of our results to the previous approaches and discussion are given in Section V. Section VI summarizes the results. An Appendix contains some details of the approach which gives the expression for integrals over the spectral density in terms of the chain representation of continuous fraction.

Part of our results were presented in Brief Reports [24]. The paper presented here gives further results as well as the description of the new method and more details about the calculations. 


\section{EFFECTIVE HAMILTONIAN AND SMALL POLARON GREEN'S FUNCTION}

Following Refs. 99:10,15] we adopt the Hamiltonian that corresponds to one hole problem in the $\mathrm{CuO}_{2}$ plane in HTSC:

$$
\hat{H}=\tau \sum_{\mathbf{r}, \mathbf{a}_{1}, \mathbf{a}_{2}, \sigma, \sigma^{\prime}} c_{\mathbf{r}+\mathbf{a}_{1}, \sigma}^{\dagger} c_{\mathbf{r}+\mathbf{a}_{2}, \sigma^{\prime}}\left(\frac{1}{2} \delta_{\sigma \sigma^{\prime}}+2 \vec{S}_{\sigma \sigma^{\prime}} \vec{S}_{\mathbf{r}}\right)+\frac{J}{2} \sum_{\mathbf{r}, \mathbf{g}} \vec{S}_{\mathbf{r}} \vec{S}_{\mathbf{r}+\mathbf{g}}
$$

$\mathbf{a}_{1}, \mathbf{a}_{2}= \pm \frac{1}{2} \mathbf{g}_{x}, \pm \frac{1}{2} \mathbf{g}_{y}, \mathbf{g}= \pm \mathbf{g}_{x}, \pm \mathbf{g}_{y}$

Here and below $\mathbf{g}_{x, y}$ are basic vectors of a copper square lattice $(|\mathbf{g}| \equiv 1), \mathbf{r}+\mathbf{a}$ are four vectors of $\mathrm{O}$ sites nearest to the $\mathrm{Cu}$ site $\mathbf{r}$, the operator $c_{\sigma}^{\dagger}$ creates a hole with the spin index $\sigma= \pm 1$ at the $\mathrm{O}$ site, $\vec{s}_{\sigma \sigma^{\prime}}=\frac{1}{2} \vec{\sigma}_{\sigma \sigma^{\prime}}$, operator $\mathbf{S}$ represents the localized spin on the copper site. As mentioned above $\tau$ is the integral of oxygen hole hoppings that takes into account the coupling of the hole motion with copper spin subsystem. $J$ is the constant of nearest neighbor AFM exchange between the copper spins.

It is well known that the most prominent feature of the Hamiltonian (2) is that the low-energy physics of hole excitations is described by the Bloch sums $\mathcal{B}_{\mathbf{k}, \sigma}^{\dagger}$ based on one site small polaron operators $\mathcal{B}_{\mathbf{r} \sigma}^{\dagger}$

$$
\begin{gathered}
\mathcal{B}_{\mathbf{k}, \sigma}^{\dagger}=\frac{1}{\sqrt{N K_{\mathbf{k}}}} \sum_{\mathbf{r}} \mathrm{e}^{i \mathbf{k} \cdot \mathbf{r}} \mathcal{B}_{\mathbf{r}, \sigma}^{\dagger}, \\
\mathcal{B}_{\mathbf{r}, \sigma}^{\dagger}=\frac{1}{2} \sum_{\mathbf{a}}\left(c_{\mathbf{r}+\mathbf{a}, \sigma}^{\dagger} Z_{\mathbf{r}}^{\bar{\sigma} \bar{\sigma}}-c_{\mathbf{r}+\mathbf{a}, \bar{\sigma}}^{\dagger} Z_{\mathbf{r}}^{\sigma \bar{\sigma}}\right), \\
K_{\mathbf{k}}=\left\langle\frac{1}{N} \sum_{\mathbf{r}, \mathbf{r}^{\prime}} \mathrm{e}^{-\imath \mathbf{k}\left(\mathbf{r}-\mathbf{r}^{\prime}\right)}\left\{\mathcal{B}_{\mathbf{r}, \sigma}, \mathcal{B}_{\mathbf{r}^{\prime}, \sigma}^{\dagger}\right\}\right\rangle=1+\left(C_{\mathbf{g}}+\frac{1}{4}\right) \gamma_{\mathbf{k}} .
\end{gathered}
$$

Here $\{\},,[$,$] stand for an anticommutator and commutator, respectively; \langle\ldots\rangle \equiv$ $Q^{-1} S p\left[\mathrm{e}^{-\beta H} \ldots\right], \quad Q=S p \mathrm{e}^{-\beta H} . S p$ implies taking the trace of an operator, $\beta=(k T)^{-1}$ is an inverse temperature; $\bar{\sigma} \equiv-\sigma ; Z_{\mathbf{r}}^{\sigma_{1} \sigma_{2}} \equiv\left|\sigma_{1}\right\rangle\left\langle\sigma_{2}\right|$ are the Hubbard projection operators for $\mathrm{Cu}$ sites states; $\gamma_{\mathbf{k}}=\frac{1}{4} \sum_{\mathbf{g}} \exp (\imath \mathbf{k} \cdot \mathbf{g})$.

To calculate the average for commutators and anticommutators such as $K_{\mathbf{k}}$, we take into account that these expressions are reduced to the two-site or three-site averages of the 
Hubbard operators located at different copper sites. In the spherically symmetric approach adopted here, all these averages can be expressed in terms of the two-site spin correlation functions $C_{\mathbf{r}}=\left\langle\mathbf{S}_{0} \cdot \mathbf{S}_{\mathbf{r}}\right\rangle$. Let us recall that due to the spherical symmetry $\left\langle S_{i}^{\alpha} S_{j}^{\beta}\right\rangle=$ $\delta_{\alpha \beta}\left\langle S_{i}^{\alpha} S_{j}^{\alpha}\right\rangle=\frac{1}{3}\left\langle\mathbf{S}_{i} \cdot \mathbf{S}_{j}\right\rangle,\left\langle S_{i}^{\alpha}\right\rangle=0$. Simultaneously, at $T \rightarrow 0$ the spin subsystem is described by a long range order state with finite effective magnetization $m, C_{\mathbf{r}}(|\mathbf{r}| \rightarrow \infty)=$ $m^{2}(-1)^{r_{x}+r_{y}}$, the value of $m$ is dictated by the Bose condensation of spin excitations at the antiferromagnetic vector $\mathbf{q}_{0}=(\pi, \pi)$.

Note, that $\mathcal{B}_{\mathbf{k}, \sigma}^{\dagger}|L\rangle$ corresponds to the $\mathrm{CuO}_{2}$ plane state with the total spin equal to $\frac{1}{2}$ if $|L\rangle$ is the singlet state. We treat $\mathcal{B}_{k, \sigma}^{\dagger}$ as a candidate for the elementary excitations operator and calculate the corresponding two-time retarded Green's function $G(\mathbf{k}, \omega)$ and spectral density

$$
\begin{gathered}
A_{p}(\mathbf{k}, \omega)=-\frac{1}{\pi} \operatorname{Im} G\left(\mathbf{k}, \omega+\imath 0^{+}\right) \\
G(\mathbf{k}, \omega)=\left\langle\mathcal{B}_{\mathbf{k}, \sigma} \mid \mathcal{B}_{\mathbf{k}, \sigma}^{\dagger}\right\rangle_{\omega} \equiv-\imath \int_{t^{\prime}}^{\infty} d t e^{\imath \omega\left(t-t^{\prime}\right)}\left\langle\left\{\mathcal{B}_{\mathbf{k}, \sigma}(t), \mathcal{B}_{\mathbf{k}, \sigma}^{\dagger}\left(t^{\prime}\right)\right\}\right\rangle .
\end{gathered}
$$

The Dyson's equation for $G(\mathbf{k}, \omega)$ has the form

$$
G^{-1}(\mathbf{k}, \omega)=G_{0}^{-1}-\Sigma(\mathbf{k}, \omega), \Sigma(\mathbf{k}, \omega)=\left\langle\mathcal{R}_{\mathbf{k}, \sigma} \mid \mathcal{R}_{\mathbf{k}, \sigma}^{\dagger}\right\rangle^{(i r r)}
$$

where

$$
\begin{gathered}
G_{0}=\left(\omega-\Omega_{\mathbf{k}}\right)^{-1}, \mathcal{R}_{\mathbf{k}, \sigma}=\left[\mathcal{B}_{\mathbf{k}, \sigma}, \hat{H}\right]=\frac{1}{\sqrt{N K_{\mathbf{k}}}} \sum_{\mathbf{r}} \mathrm{e}^{-\imath \mathbf{k} \cdot \mathbf{r}} \mathcal{R}_{\mathbf{r}, \sigma} \\
\mathcal{R}_{\mathbf{r}, \sigma}=-4 \tau \mathcal{B}_{\mathbf{r}, \sigma}+\mathcal{R}_{\mathbf{r}, \sigma}^{\tau}+\mathcal{R}_{\mathbf{r}, \sigma}^{J} \\
\mathcal{R}_{\mathbf{r}, \sigma}^{\tau}=-\frac{\tau}{2} \sigma\left(\sum_{\mathbf{g}, \mathbf{a}, \sigma_{1}} \sigma_{1} Z_{\mathbf{r}}^{\bar{\sigma} \sigma_{1}} c_{\mathbf{r}+\mathbf{g}+\mathbf{a}, \bar{\sigma}_{1}}-\sum_{\mathbf{g}, \mathbf{a}, \sigma_{1}, \sigma_{2}} \sigma_{2} Z_{\mathbf{r}}^{\bar{\sigma} \sigma_{1}} Z_{\mathbf{r}+\mathbf{g}}^{\sigma_{1} \sigma_{2}} c_{\mathbf{r}+\mathbf{g}+\mathbf{a}, \bar{\sigma}_{2}}\right) \\
\mathcal{R}_{\mathbf{r}, \sigma}^{J}=\frac{J}{4} \sigma\left(\sum_{\mathbf{g}, \mathbf{a}, \sigma_{1}} \sigma_{1}\left(Z_{\mathbf{r}}^{\bar{\sigma} \sigma_{2}} Z_{\mathbf{r}+\mathbf{g}}^{\sigma_{2} \bar{\sigma}_{1}}-Z_{\mathbf{r}+\mathbf{g}}^{\bar{\sigma} \sigma_{2}} Z_{\mathbf{r}}^{\sigma_{2} \bar{\sigma}_{1}}\right) c_{\mathbf{r}+\mathbf{a}, \sigma_{1}}\right) \\
\Omega_{\mathbf{k}}=\left\langle\left\{\mathcal{R}_{\mathbf{k}, \sigma}, \mathcal{B}_{\mathbf{k}, \sigma}^{\dagger}\right\}\right\rangle=\left(\tau Q_{\tau}+J Q_{J}\right) / K_{\mathbf{k}}, \\
Q_{\tau}(\mathbf{k})=-\frac{7}{2}-8\left(\frac{1}{4}+C_{\mathbf{g}}\right) \gamma_{\mathbf{k}}+\left(\frac{1}{8}-C_{\mathbf{g}}+\frac{1}{2} C_{2 \mathbf{g}}\right) \gamma_{2 \mathbf{k}}+2\left(\frac{1}{8}-C_{\mathbf{g}}+\frac{1}{2} C_{\mathbf{d}}\right) \gamma_{\mathbf{d k}},
\end{gathered}
$$




$$
\begin{gathered}
Q_{J}(\mathbf{k})=C_{\mathbf{g}}\left(\gamma_{\mathbf{k}}-4\right) \\
\langle\mathcal{R} \mid \mathcal{R}\rangle^{(i r r)}=\left\langle\mathcal{R}_{\mathbf{k}, \sigma} \mid \mathcal{R}_{\mathbf{k}, \sigma}^{\dagger}\right\rangle-\left\langle\mathcal{R}_{\mathbf{k}, \sigma} \mid \mathcal{B}_{\mathbf{k}, \sigma}^{\dagger}\right\rangle \frac{1}{\left\langle\mathcal{B}_{\mathbf{k}, \sigma} \mid \mathcal{B}_{\mathbf{k}, \sigma}^{\dagger}\right\rangle}\left\langle\mathcal{B}_{\mathbf{k}, \sigma} \mid \mathcal{R}_{\mathbf{k}, \sigma}^{\dagger}\right\rangle .
\end{gathered}
$$

Here and below $\mathbf{d}=\mathbf{g}_{x}+\mathbf{g}_{y}, \gamma_{\mathbf{d k}}=\cos \left(k_{x} g\right) \cos \left(k_{y} g\right)$.

We see from Eqs.(16) and (11), that the self-energy $\Sigma(\mathbf{k}, \omega)$ accounting for interaction effects is expressed through the higher-order Green's functions. One should notice, first, that the terms linear in $\mathcal{B}_{\mathbf{k}, \sigma}$ do not contribute to the irreducible Green's function (11). Second, the lowest-order self-energy contribution is provided by the first term in the right-hand side of the expression (11), while the second term leads to higher-order corrections. Following Ref. [7] we evaluate (11) with a proper decoupling procedure for two time correlation function $\left\langle\mathcal{R}_{\mathbf{k}, \sigma}(t) \mathcal{R}_{\mathbf{k}, \sigma}^{\dagger}\left(t^{\prime}\right)\right\rangle$. This procedure is equivalent to the self-consistent Born approximation in a usual diagrammatic technique [7]. In our case this means that the two-time correlation function is decoupled into the spin-spin correlation function and the polaron-polaron correlation function. The adopted decoupling procedure preserves the main character of polaron site operator (4) - four hole site operators surround the copper spin operator. It schematically has the form

$$
\begin{gathered}
\left\langle Z_{\mathbf{r}_{1}}(t)\left(\sum_{\mathbf{a}_{1}} c_{\mathbf{r}_{2}+\mathbf{a}_{1}}(t) Z_{\mathbf{r}_{2}}(t)\right)\left(\sum_{\mathbf{a}_{2}} Z_{\mathbf{r}_{3}}\left(t^{\prime}\right) c_{\mathbf{r}_{3}+\mathbf{a}_{2}}^{\dagger}\left(t^{\prime}\right)\right) Z_{\mathbf{r}_{4}}\left(t^{\prime}\right)\right\rangle \simeq \\
\left\langle\left(\sum_{\mathbf{a}_{1}} c_{\mathbf{r}_{2}+\mathbf{a}_{1}}(t) Z_{\mathbf{r}_{2}}(t)\right)\left(\sum_{\mathbf{a}_{2}} Z_{\mathbf{r}_{3}}\left(t^{\prime}\right) c_{\mathbf{r}_{3}+\mathbf{a}_{2}}^{\dagger}\left(t^{\prime}\right)\right)\right\rangle\left\langle Z_{\mathbf{r}_{1}}(t) Z_{\mathbf{r}_{4}}\left(t^{\prime}\right)\right\rangle .
\end{gathered}
$$

Let us mention that we also tested the more complex decoupling procedure, and it did not qualitatively alter the results given by approximation (12). On the next step we project polaron operators in (12) onto $\mathcal{B}_{\mathrm{k} \sigma}$ :

$$
c_{i}(t) Z_{j}(t) \simeq \xi \mathcal{B}_{\mathbf{k} \sigma}(t), \xi=\left\langle\left\{c_{i}(t) Z_{j}(t), \mathcal{B}_{\mathbf{k}, \sigma}^{\dagger}\right\}\right\rangle .
$$

Taking into account, that we shall calculate only the irreducible part of Green's function (11), the averages $\left\langle Z_{\mathbf{r}_{1}}(t) Z_{\mathbf{r}_{4}}\left(t^{\prime}\right)\right\rangle$ are transformed to corresponding spin-spin correlation functions $\left\langle S_{\mathbf{r}_{1}}^{\alpha}(t) S_{\mathbf{r}_{4}}^{\alpha}\left(t^{\prime}\right)\right\rangle$. Collecting all terms, we have 


$$
\begin{gathered}
\left\langle\mathcal{R}_{\mathbf{k}, \sigma}(t) \mathcal{R}_{\mathbf{k}, \sigma}^{\dagger}\left(t^{\prime}\right)\right\rangle \simeq N^{-1} \sum_{\mathbf{q}} \frac{K_{\mathbf{k}-\mathbf{q}}}{K_{\mathbf{k}}} \Gamma^{2}(\mathbf{k}, \mathbf{q})\left\langle\mathcal{B}_{\mathbf{k}-\mathbf{q}, \sigma}(t) \mathcal{B}_{\mathbf{k}-\mathbf{q}, \sigma}^{\dagger}\left(t^{\prime}\right)\right\rangle\left\langle\mathbf{S}_{-\mathbf{q}}(t) \mathbf{S}_{\mathbf{q}}\left(t^{\prime}\right)\right\rangle \\
\Gamma(\mathbf{k}, \mathbf{q})=\tau \Gamma_{\tau}(\mathbf{k}, \mathbf{q})+\frac{J}{2} \Gamma_{J}(\mathbf{k}, \mathbf{q}) \\
\Gamma_{\tau}(\mathbf{k}, \mathbf{q})=4 \gamma_{\mathbf{k}-\mathbf{q}}\left[\left(1+\gamma_{\mathbf{k}-\mathbf{q}}\right) / 2 K_{\mathbf{k}-\mathbf{q}}-1\right] \\
\Gamma_{J}(\mathbf{k}, \mathbf{q})=4 \gamma_{\mathbf{q}}\left[\left(\frac{3}{4}-C_{\mathbf{g}}\right) \frac{4 \gamma_{\mathbf{k}-\mathbf{q}}}{3 K_{\mathbf{k}-\mathbf{q}}}-1\right] \\
\left\langle\mathbf{S}_{-\mathbf{q}}(t) \mathbf{S}_{\mathbf{q}}\left(t^{\prime}\right)\right\rangle=\frac{1}{N} \sum_{\mathbf{r}, \mathbf{r}^{\prime}} e^{i \mathbf{q} \cdot\left(\mathbf{r}^{\prime}-\mathbf{r}\right)}\left\langle\mathbf{S}_{\mathbf{r}}(t) \mathbf{S}_{\mathbf{r}^{\prime}}\left(t^{\prime}\right)\right\rangle
\end{gathered}
$$

Using the spectral representation for Green's functions we obtain the following intermediate result for the self-energy

$$
\begin{gathered}
\Sigma(\mathbf{k}, \omega)=N^{-1} \sum_{\mathbf{q}} \frac{K_{\mathbf{k}-\mathbf{q}}}{K_{\mathbf{k}}} \Gamma^{2}(\mathbf{k}, \mathbf{q}) \int_{-\infty}^{\infty} \frac{d \omega_{1}}{\pi} \times \\
\int_{-\infty}^{\infty} \frac{d \omega_{2}}{\pi} \frac{\mathrm{e}^{\beta\left(\omega_{1}+\omega_{2}\right)}+1}{\left(\mathrm{e}^{\beta \omega_{1}}+1\right)\left(\mathrm{e}^{\beta \omega_{2}}-1\right)} \frac{\operatorname{Im}\left[G\left(\mathbf{k}-\mathbf{q}, \omega_{1}+\imath 0^{+}\right)\right] \operatorname{Im}\left[D\left(\mathbf{q}, \omega_{2}+\imath 0^{+}\right)\right]}{\omega-\left(\omega_{1}+\omega_{2}\right)+\imath 0^{+}} .
\end{gathered}
$$

Here the spin excitation Green's function [22,23]

$$
\begin{gathered}
D(\mathbf{q}, \omega)=\left\langle S_{-\mathbf{q}}^{z} \mid S_{\mathbf{q}}^{z}\right\rangle=-\frac{8 J C_{\mathbf{g}}}{3} \frac{1-\gamma_{\mathbf{q}}}{\omega^{2}-\omega_{\mathbf{q}}^{2}} \\
\omega_{\mathbf{q}}^{2}=-32 J \alpha_{1} C_{\mathbf{g}}\left(1-\gamma_{\mathbf{q}}\right)\left(2 \Delta+1+\gamma_{\mathbf{q}}\right)
\end{gathered}
$$

we neglect the influence of doped holes on copper spin dynamics and take the spin spectrum parameters calculated in Ref. [22] (the vertex correction $\alpha_{1}=1.7$, the spin excitations condensation part $\left.m^{2}=0.0225\right)$.

As a result we come to the integral equation for the Green's function that always arises within the framework of SCBA

$$
G(\mathbf{k}, \omega)=\frac{1}{\omega-\Omega_{\mathbf{k}}-\Sigma(\mathbf{k}, \omega)}
$$

where

$$
\Sigma(\mathbf{k}, \omega)=N^{-1} \sum_{\mathbf{q}} M^{2}(\mathbf{k}, \mathbf{q})\left[\left(1+\nu_{\mathbf{q}}\right) G\left(\mathbf{k}-\mathbf{q}, \omega-\omega_{\mathbf{q}}\right)+\nu_{\mathbf{q}} G\left(\mathbf{k}-\mathbf{q}, \omega+\omega_{\mathbf{q}}\right)\right]
$$


$\nu_{\mathbf{q}}=1 /\left[\exp \left(\beta \omega_{\mathbf{q}}\right)-1\right]$ is the Bose function,

$$
M^{2}(\mathbf{k}, \mathbf{q})=\frac{K_{\mathbf{k}-\mathbf{q}}}{K_{\mathbf{k}}} \Gamma^{2}(\mathbf{k}, \mathbf{q}) \frac{\left(-4 C_{\mathbf{g}}\right)\left(1-\gamma_{\mathbf{q}}\right)}{\omega_{\mathbf{q}}} .
$$

$\Gamma(\mathbf{k}, \mathbf{q})$ corresponds to the bare vertex for the coupling between a spin polaron and a spin wave. It is known [25] that this vertex is substantially renormalized for $\mathbf{q}$ close to the AFM vector $\mathbf{q}_{0}=(\pi, \pi)$. This renormalization is due to the strong interaction of a polaron with the condensation part of spin excitations that must be taken into account from the very beginning. As a result, the renormalized vertex $\tilde{\Gamma}(\mathbf{k}, \mathbf{q})$ must be proportional to 25 $\left[\left(\mathbf{q}-\mathbf{q}_{0}\right)^{2}+L_{s}^{-2}\right]^{1 / 2}, L_{s}$ being the spin-spin correlation length, $L_{s} \rightarrow \infty$ in our case of a long range order state of the spin subsystem. Below this renormalization is taken into account empirically by the substitution

$$
\Gamma(\mathbf{k}, \mathbf{q}) \rightarrow \tilde{\Gamma}(\mathbf{k}, \mathbf{q})=\Gamma(\mathbf{k}, \mathbf{q}) \sqrt{\left(1+\gamma_{\mathbf{q}}\right)}
$$

The introduced vertex correction is proportional to $\left|\mathbf{q}-\mathbf{q}_{0}\right|$ for $\mathbf{q}$ close to $\mathbf{q}_{0}$. We have used

also the following two functions for the vertex correction $\sqrt{1+\gamma_{\mathbf{q}}^{3}}$ and $\sqrt{1+\gamma_{\mathbf{q}}^{5}}$ and have obtained the results similar to those presented below. Let us mention that the bare vertex leads the dramatic decrease of the QP bandwidth.

\section{SOLUTION OF THE INTEGRAL EQUATION}

The equation (17) is usually solved by an iteration procedure. We propose here an alternative way, based on the continued fraction expansion of $G(\mathbf{k}, z)$

$$
G(\mathbf{k}, z)=\frac{b_{0}^{2}}{z-a_{0}-} \frac{b_{1}^{2}}{z-a_{1}-} \cdots \frac{b_{n}^{2}}{z-a_{n}-} \cdots, \quad a_{n}=a_{n}(\mathbf{k}), \quad b_{n}=b_{n}(\mathbf{k})
$$

where

$$
b_{0}^{2}=\int_{-\infty}^{+\infty} A_{p}(\mathbf{k}, \omega) d \omega=K_{\mathbf{k}}, \quad a_{0}=\frac{1}{b_{0}^{2}} \int_{-\infty}^{+\infty} \omega A_{p}(\mathbf{k}, \omega) d \omega=\Omega_{\mathbf{k}} .
$$

The coefficients $b_{n}, a_{n}, n>0$ are related with the spectral density $A_{p}(\mathbf{k}, \omega)$ via the set of orthogonal polynomials $P_{n}(\omega)$, satisfying the recurrence [26 30]:

$$
P_{-1}(\omega)=0, \quad P_{0}(\omega)=1
$$




$$
P_{n+1}(\omega)=\left(\omega-a_{n}\right) P_{n}(\omega)-b_{n}^{2} P_{n-1}(\omega)
$$

and

$$
\begin{aligned}
a_{n} & =\frac{\int_{-\infty}^{+\infty} \omega P_{n}^{2}(\omega) A_{p}(\mathbf{k}, \omega) d \omega}{\int_{-\infty}^{+\infty} P_{n}^{2}(\omega) A_{p}(\mathbf{k}, \omega) d \omega}, \\
b_{n+1}^{2} & =\frac{\int_{-\infty}^{+\infty} P_{n+1}^{2}(\omega) A_{p}(\mathbf{k}, \omega) d \omega}{\int_{-\infty}^{+\infty} P_{n}^{2}(\omega) A_{p}(\mathbf{k}, \omega) d \omega} .
\end{aligned}
$$

Here we have used the nonnormalized form of the polynomials $\int_{-\infty}^{+\infty} P_{n}(\omega) P_{s}(\omega) A_{p}(\mathbf{k}, \omega) d \omega=\delta_{n s}\left(\prod_{m=1}^{m=n} b_{m}\right)^{2}$.

Comparing Eqs.(21) and (17) we see that the self energy $\Sigma(\mathbf{k}, z)$ is the continued fraction similar to $G(\mathbf{k}, z)$. Thus we can introduce the spectral density

$$
\rho(\mathbf{k}, \omega)=-\operatorname{Im}\left[\Sigma\left(\mathbf{k}, \omega+\imath 0^{+}\right)\right] / \pi
$$

and the set of polynomials $\Pi_{n}(\omega)$ with the recurrence analogous to (22):

$$
\Pi_{n}(\omega)=\left(\omega-a_{n}\right) \Pi_{n-1}(\omega)-b_{n}^{2} \Pi_{n-2}(\omega), \quad \Pi_{0}(\omega)=1, \quad \Pi_{-1}(\omega)=0,
$$

where

$$
\begin{gathered}
b_{1}^{2}=\int_{-\infty}^{\infty} \rho(\mathbf{k}, \omega) d \omega, \quad a_{1}=\frac{1}{b_{1}^{2}} \int_{-\infty}^{+\infty} \omega \rho(\mathbf{k}, \omega) d \omega \\
a_{n+1}=\frac{\int_{-\infty}^{+\infty} \omega \Pi_{n}^{2}(\omega) \rho(\mathbf{k}, \omega) d \omega}{\int_{-\infty}^{+\infty} \Pi_{n}^{2}(\omega) \rho(\mathbf{k}, \omega) d \omega}, \quad b_{n+1}^{2}=\frac{\int_{-\infty}^{+\infty} \Pi_{n}^{2}(\omega) \rho(\mathbf{k}, \omega) d \omega}{\int_{-\infty}^{+\infty} \Pi_{n-1}^{2}(\omega) \rho(\mathbf{k}, \omega) d \omega}, \quad n \geq 1 .
\end{gathered}
$$

On the other hand, we have from Eq.(18)

$$
\rho(\mathbf{k}, \omega)=\frac{1}{K_{\mathbf{k}}} N^{-1} \sum_{\mathbf{q}} M^{2}(\mathbf{k}, \mathbf{q})\left[\left(1+\nu_{\mathbf{q}}\right) A_{p}\left(\mathbf{k}-\mathbf{q}, z-\omega_{\mathbf{q}}\right)+\nu_{\mathbf{q}} A_{p}\left(\mathbf{k}-\mathbf{q}, z+\omega_{\mathbf{q}}\right)\right]
$$

If we shall put the expression for $\rho(\mathbf{k}, \omega)$ in Eq.(25) then the coefficients $a_{n+1}$ and $b_{n+2}$ will be expressed through the integrals of the form

$$
\int_{-\infty}^{\infty}\left(\omega \pm \omega_{\mathbf{q}}\right)^{m} \Pi_{i}^{2} A_{p}(\mathbf{k}-\mathbf{q}, \omega) d w, \quad i \leq n, \quad m=0,1
$$

Now, the trick is that the polynomials in $\omega$ in the integrals (27) have the degree less than or equal $2 n+1$. As it was proved by Nex [27] such integrals may be expressed through 
the coefficients $\left\{a_{0}, \ldots, a_{n}, b_{0} \ldots, b_{n}\right\}$. The details of such a procedure are presented in the Appendix. So, it turns out that in SCBA we can recursively calculate pairs of coefficients $a_{n+1}, b_{n+1}$ and to obtain $\Sigma(\mathbf{k}, \omega)$ in the continued fraction form. Of course we must calculate the coefficients at all the chosen $\mathbf{k}+\mathbf{q}$ points in the first Brillouin zone simultaneously. Below the chosen points correspond to a lattice of $32 \times 32$ unit cells. The presented procedure provides the opportunity to avoid the iterative solution of Eq.(17) for complex energies.

\section{TERMINATION OF THE CONTINUED FRACTION}

The procedure outlined in the previous section would be efficient if after calculating a finite number of coefficients $a_{n}, b_{n}, n \leq n_{0}$, we could appropriately approximate the part (infinite in our case) of the continued fraction $T_{n_{0}}$, which has not been calculated. In other words, we rewrite the expression (21) in the form

$$
G(\mathbf{k}, z)=\frac{b_{0}^{2}}{z-a_{0}-} \frac{b_{1}^{2}}{z-a_{1}-} \cdots \frac{b_{n_{0}}^{2}}{z-a_{n_{0}}-T_{n_{0}}(\mathbf{k}, z)}
$$

and try to find a function $\tilde{T}_{n_{0}}$ (so called "terminator") that is close to $T_{n_{0}}$.

Various ways to construct such approximations are described in the literature on the recursion method (see Refs. 27 29] ). The asymptotic behavior of continued fraction coefficients is governed by the band structure and singularities of spectral density [29]. The main asymptotic behavior depends on the band structure: $\left\{a_{n}\right\}$ and $\left\{b_{n}\right\}$ converge towards limits in the single band case, oscillate endlessly in a predictable way in the multiband case. Damped oscillations are created by isolated singularities. The main point here is that an isolated simple pole produces exponentially damped contribution in $\left\{a_{n}\right\},\left\{b_{n}\right\}, n \rightarrow \infty$. For our case it means that the quasiparticle pole position and weight could be obtained with the high accuracy from finite number of coefficients, and the asymptotic behavior determines the incoherent part of the spectrum. It is obvious that the spectrum we deal with has an lower bound and no upper bound. We can thus expect that coefficients will not converge to some finite values but will grow up to infinity. 
In Fig.11 we represent the coefficients $a_{n}, b_{n}$ as functions on $n$ calculated according the procedure described in the preceding section for two values of $J, J=0.7 \tau$ and $J=0.1 \tau$ and for $\mathbf{k}=(\pi / 2, \pi / 2)$. As it may be seen, the distinctive feature of this dependence is that for large $n$ the coefficients $a_{n}, b_{n}$ are linear functions of $n$. For all this, the slope for $a_{n}$ coefficients is twice as many as the slope for $b_{n}$. So the coefficients behavior may be approximated as

$$
b_{n} \approx \lambda_{1} n+\lambda_{2}, \quad a_{n} \approx 2 \lambda_{1} n+\lambda_{3}, \quad \lambda_{i}=\lambda_{i}(\mathbf{k}), \quad n \gg 1
$$

It is interesting that the analogous behavior show the coefficients for $t-J$ model approximated by slave-fermion Hamiltonian [2] treated within the self-consistent Born approximation $\left[4\right.$. For $J=0.4 t, \mathbf{k}=(\pi / 2, \pi / 2)$ the coefficients $a_{n}, b_{n}$ dictated by the relation analogous to (18) are represented in Fig. 8 (a).

Now we shall show that the same asymptotic (29) has the CF expansion of incomplete Gamma function that is written as [31]

$$
\Gamma(\alpha, x)=\frac{\mathrm{e}^{-x} x^{\alpha}}{x+} \frac{1-\alpha}{1+} \frac{1}{x+} \frac{2-\alpha}{1+} \cdots
$$

This circumstance we shall use for the construction of the terminator $\tilde{T}_{N}(\mathbf{k}, z)$ for $G(\mathbf{k}, z)$ (28)

Let us introduce the function

$$
\tilde{g}(\alpha, x)=-\frac{\Gamma(\alpha,-x)}{\mathrm{e}^{x}(-x)^{\alpha}}=1 /\left(x-\frac{1-\alpha}{1-\theta_{1}}\right)
$$

where

$$
\theta_{n}=n /\left(x-\frac{n+1-\alpha}{1-\theta_{n+1}}\right)
$$

In order to rewrite the CF (31) in the form analogous to Eq.(21) we denote

$$
\frac{1}{1-\theta_{n}} \equiv 1+n t_{n}
$$

Then it is not difficult to obtain the relations 


$$
t_{n}=\frac{1}{x-(2 n+1-\alpha)-(n+1)(n+1-\alpha) t_{n+1}}
$$

so that

$$
\tilde{g}(\alpha, x)=t_{0}
$$

has the form (28) with the coefficients $\tilde{b}_{0}^{2}=1$ and

$$
\tilde{a}_{n}=2 n+1-\alpha, \quad \tilde{b}_{n}^{2}=n(n-\alpha) .
$$

Comparing Eqs. (29) and (36) for large $n$, when $\sqrt{n(n-\alpha)} \approx n-\alpha / 2$, we see that the substitution

$$
\alpha=-\frac{2 \lambda_{2}}{\lambda_{1}}, x=\frac{z+2 \lambda_{2}-\lambda_{3}+\lambda_{1}}{\lambda_{1}}
$$

leads to the function $\tilde{G}$

$$
\tilde{G}(\mathbf{k}, z)=\frac{1}{\lambda_{1}} \tilde{g}\left(-\frac{2 \lambda_{2}}{\lambda_{1}}, \frac{z+2 \lambda_{2}-\lambda_{3}+\lambda_{1}}{\lambda_{1}}\right)
$$

that has the same asymptotic as $G(\mathbf{k}, z)$ (21). It means that $\tilde{G}(\mathbf{k}, z)$ can be used as the terminator for $G(\mathbf{k}, z)$. , i.e. we can express $\tilde{T}_{n_{0}}(\mathbf{k}, z)=\tilde{b}_{n_{0}+1} t_{n_{0}+1} \operatorname{through} \tilde{G}(\mathbf{k}, z)$, and the coefficients $\tilde{a}_{n}, \tilde{b}_{n}, n \leq n_{0}$, and then substitute it for $T_{n_{0}}(\mathbf{k}, z)$ (see [28] for the details of matching Greenians).

We thus obtain $G(\mathbf{k}, z)$ in the whole complex energy plane including the real axis. Note that usually the procedure of discretizing the energy range $\omega$ is used for iteration process when the Dyson's equation is solved numerically. It is not obvious that such a self-consistent solution leads to the correct analytical properties of the resulting Green's function. In contrast the continued fraction representation guarantees these properties (e.g. the positive definiteness of spectral function $\left.A_{p}(\mathbf{k}, \omega)\right)$.

\section{RESULTS AND DISCUSSION}

In this section we represent our results for the retarded Green's function $G(\mathbf{k}, \omega)$ for three-band model at $T=0$. The self consistent equation (17) was solved on a $32 \times 32$ cell lattice. The number of calculated CF levels $n_{0}$ was taken $n_{0}=30$. 
To begin with, we check the validity of the method outlined above by calculating the spinless hole Green's function for the $t-J$ model and compare the results with the results of Martinez and Horsch [4] obtained by usual iteration procedure. In Fig.? $A_{h}\left(\mathbf{k}_{1}, \omega+\right.$

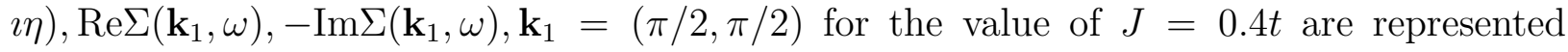
for the $16 \times 16$ site lattice and broadening constant $\eta=0.01 t$. The comparison of Fig.2 (b),(c),(d) and the corresponding functions given by Fig.7,8 from Ref. 四 (the same lattice size and $\eta$ ) demonstrates that the position of peaks of the hole spectral function and their intensities coincide. Some difference is that our $A_{h}\left(\mathbf{k}_{1}, \omega\right)$, is more smooth and there are no strong oscillations in our self energy $\Sigma(\mathbf{k}, \omega)$ in the interval $(-2 t<\omega<-0.75 t)$.

The results for the small spin polaron spectral density, real and imaginary parts of the self energy for the characteristic value of energetic parameter $J=0.7 \tau$ are given in Fig. 3 for the symmetrical points $\mathbf{k}_{1}=(\pi / 2, \pi / 2), \mathbf{k}_{2}=(0,0), \mathbf{k}_{3}=(\pi, \pi)$. The energy broadening parameter is $\eta=0.002$ (we will refer all quantities in units of $\tau$ from now on). We find main common feature in the spectral density for $\mathbf{k}_{1}$ and $\mathbf{k}_{2}$ : a sharp quasiparticle peak exists at the bottom of each spectrum. The position of the QP peak corresponds to the condition $\operatorname{Re} G^{-1}(\mathbf{k}, \omega)=0$, i.e., the point where we have the crossing of the functions $y=\omega-\Omega_{\mathbf{k}}$ and $\operatorname{Re} \Sigma(\mathbf{k}, \omega)$, see Figs. 3 (a) and 3 (b). In Fig. 3 (d) we show $A_{p}\left(\mathbf{k}_{1}, \omega\right)$ calculated for $\eta=0.002$ (solid line) and $\eta=0.0005$ (dashed line) in order to study the scaling behavior of the peaks and their widths with respect to changes in $\eta$. Both peaks fit quite closely with a Lorentzian $(1 / \pi)\left\{Z\left(\mathbf{k}_{1}\right) \eta /\left[\left(\omega-\epsilon\left(\mathbf{k}_{1}\right)\right)^{2}+\eta^{2}\right]\right\}, \epsilon\left(\mathbf{k}_{1}\right)$ is the location of the peak, which in the limit $\eta \rightarrow 0$ becomes $Z\left(\mathbf{k}_{1}\right) \delta\left(\omega-\epsilon\left(\mathbf{k}_{1}\right)\right)$. It means that $\operatorname{Im} \Sigma\left(\mathbf{k}, \omega_{p}\right) \rightarrow 0$ in the same limit. Here and below we shall speak about the position $\epsilon(\mathbf{k})$ of such peaks (with imaginary part of pole close to zero) as about the energy of the quasiparticle.

Figs. 3 (a) and 3 (b) also demonstrate that the incoherent part of $A_{p}(\mathbf{k}, \omega)$ increases and the pole strength decreases with the increase of $\epsilon(\mathbf{k}), Z\left(\mathbf{k}_{1}\right)=0.82, Z\left(\mathbf{k}_{2}\right)=0.347$. Let us recall that $\Omega_{\mathrm{k}}$ represents the center of gravity of the spectral function. In our Figures the center of gravity corresponds to the crossing of the real axis by the line $y=\omega-\Omega_{\mathbf{k}}$. So, if the quasiparticle peak is far from this point we would have a great incoherent part. 
Quite different features demonstrate $A_{p}\left(\mathbf{k}_{3}, \omega\right)$ in Fig. 3 (c). The broad lowest peak is determined by the appearance of nonzero $\operatorname{Im} \Sigma\left(\mathbf{k}_{3}, \omega\right)$ in the region where $\operatorname{Re} G^{-1}\left(\mathbf{k}_{3}, \omega\right)$ has no zeroes. Two broad additional peaks at $\omega \approx-1.6$ and $\omega \approx-1.05$ are formed due to the zero values of $\operatorname{Re} G^{-1}\left(\mathbf{k}_{3}, \omega\right)$ close to these $\omega$. But at the same time the $\operatorname{Im} \Sigma\left(\mathbf{k}_{3}, \omega\right)$ is strong in these regions. Moreover, the maximum of $\operatorname{Im} \Sigma\left(\mathbf{k}_{3}, \omega\right)$ (near the point $\omega \approx-1.37$ ) determines the local minimum of $A_{p}\left(\mathbf{k}_{3}, \omega\right)$ despite of the fact, that this point is close to the frequency value where $\operatorname{Re} G^{-1}\left(\mathbf{k}_{3}, \omega\right)=0$. It is clear that it is impossible to treat any of $A_{p}\left(\mathbf{k}_{3}, \omega\right)$ peaks as a quasiparticle one. Let us mind that the qualitative behavior of the real part of the self-energy in Fig. 3 (c) is close to that one which is represented by Kampf and Schrieffer, see [32 Fig.3 (b), for the pseudogap regime of the Hubbard model. Fig.3 (c) demonstrate three solutions of $\operatorname{Re} G^{-1}\left(\mathbf{k}_{3}, \omega\right)=0$. Although there is a sharp crossover from a situation with three solutions to one quasiparticle solution, the spectral function still changes smoothly due to the presence of imaginary part of $\Sigma$.

Figs. 3 (b) and (c) demonstrate qualitatively different character of $A_{p}(\mathbf{k}, \omega)$ for the points $\mathbf{k}_{2}=(0,0), \mathbf{k}_{3}=(\pi, \pi)$. This is the consequence of our spherically symmetric approach for treating the AFM copper spin subsystem. As mentioned in Introduction this approach gives rise to the spectral function periodicity relative to the full Brillouin zone, not magnetic one.

In Fig. (1 we show the dispersion relation $\epsilon(\mathbf{k})$ of the quasiparticle band and mean field dispersion $\Omega_{\mathbf{k}}$ along symmetry lines in the Brillouin zone. For $\epsilon(\mathbf{k})$ we reproduce only that $\mathbf{k}$ - values for which the lowest peak has a pronounced quasiparticle peak taking the following criteria: $-\operatorname{Im} \Sigma(\mathbf{k}, \epsilon(\mathbf{k})+\imath \eta)<2 \eta, \eta=0.002$. As it is known [15], due to the antiferromagnetic character of spin correlation functions the $\Omega_{\mathbf{k}}$ demonstrates a "flat dispersion region" close to the line $\gamma_{\mathbf{k}}<0,\left|\gamma_{\mathbf{k}}\right| \ll 1$,i.e. close to the boundary of magnetic Brillouin zone X-N-X, see Fig. 4. As it may be seen from Fig. 4 the quasiparticle band exists for greater part of the Brillouin zone except the region of the top of the $\Omega_{\mathbf{k}}$ spectrum. Moreover, the dispersion law $\epsilon(\mathbf{k})$ qualitatively reproduces main features of the spectrum $\Omega_{\mathbf{k}}$. As it is mentioned in the Introduction, $\Omega_{\mathbf{k}}$ demonstrates the important features of the hole spectrum for $\mathrm{CuO}_{2}$ plane if one takes into account O-O hoppings and spin frustration [21]. We hope that in this 
case $\epsilon(\mathbf{k})$ will reproduce these features also.

Let us compare the small polaron spectral density $A_{p}(\mathbf{k}, \omega)$ with the results for the bare hole $A_{h}(\mathbf{k}, \omega)$ given by Kabanov and Vagov 12 for $\mathbf{k}_{1}=(\pi / 2, \pi / 2), J=0.7 \tau$, see Fig. 3 (a). First, Fig. 3 (a) indicates that $A_{p}\left(\mathbf{k}_{1}, \omega\right)$ demonstrates much sharper QP peaks relative to the results for a bare hole. For example, the pole strength $Z_{p}\left(\mathbf{k}_{1}\right)$ for the QP peak of $A_{p}(\mathbf{k}, \omega)$ equals to $Z_{p}(\mathbf{k})=0.82$. The corresponding value for $A_{h}$ given by Ref. 12 is much smaller, $Z_{h}(\mathbf{k})=0.25$.

Secondly, Fig. 3 (a) explicitly demonstrates the one peak structure $A_{p}\left(\mathbf{k}_{1}, \omega\right)$ in contrast to $A_{h}\left(\mathbf{k}_{1}, \omega\right)$. Finally, it is important, that the bottom of our QP $\epsilon(\mathbf{k})=-3.52$ is substantially lower than $\omega_{\min , h}=-2.6$ from [12]. These results are the consequence of the fact, that elementary excitation - spin polaron of small radii $\mathcal{B}_{\mathbf{k}, \sigma}$ - from the beginning takes into account the strong local hole-spin coupling.

It is clear that the QP peaks for a bare hole and a small polaron have to coincide in the exact solution of the problem. The mentioned above discrepancies between our calculations and that one of Ref. [12] are the consequence of different approximations.

In order to test the convergence of our results relative to the increase of the lattice size and the number of calculated CF levels $N L$, in Fig. 5 we show the QP peak of $A_{p}(\mathbf{k}, \omega)$ at $\mathbf{k}=(\pi / 2, \pi / 2), J=0.7$ for different lattices and $n_{0}$. This peak, as it is seen from Fig.5 (a) and (b), changes insignificantly in going from $24 \times 24$ to $32 \times 32$ cell lattice and from 22 to $30 N L$.

We consider now the transformation of $G(\mathbf{k}, \omega)$ with the decrease of $J$. In order to clarify how the character of $A_{p}(\mathbf{k}, \omega)$ peaks is changed, in Fig.6 we show $A_{p}(\mathbf{k}, \omega)$ for the value of $J=0.1$ at points $\mathbf{k}_{1}=(\pi / 2, \pi / 2), \mathbf{k}_{2}=(0,0), \mathbf{k}_{3}=(\pi, \pi)$. The decrease of $J$ leads to the enlargement of broad incoherent part of $A_{p}(\mathbf{k}, \omega)$.

As before the flat band region of the QP band bottom enlarges along a magnetic Brillouin zone boundary. It is represented by the point $\mathbf{k}_{1}$. In Fig.6 (a) $A_{p}\left(\mathbf{k}_{1}, \omega\right)$ demonstrates explicitly a rather strong QP peak, $Z_{p}\left(\mathbf{k}_{1}\right)=0.5$, which corresponds to the condition $\operatorname{Re} G^{-1}(\mathbf{k}, \omega)=0$. Quite different character of $A_{p}(\mathbf{k}, \omega)$ is typical for $\mathbf{k}$ that correspond to 
the tops of $\Omega_{\mathbf{k}}$ band: in the low-energy sector for $\mathbf{k}_{2}, \mathbf{k}_{3}$, see Fig.6 (b),(c), one observes $A_{p}(\mathbf{k}, \omega)$ - peaks with small intensity. For example, the pole strength $Z_{p}$ of such a QP peak for $A_{p}\left(\mathbf{k}_{2}, \omega\right)$ equals to $Z_{p}\left(\mathbf{k}_{2}\right)=0.016$. Taking $\omega_{l}(\mathbf{k})$ as the value of $\omega$ corresponding to the center of these lowest by energy peaks one can see that $\operatorname{Re} G^{-1}\left(\mathbf{k}, \omega_{l}(\mathbf{k}) \neq 0\right.$ for the $\mathbf{k}$ under discussion. Figs. 6 (b) and (c) demonstrates that these peaks are determined by the peaks in $\operatorname{Im} \Sigma(\mathbf{k}, \omega)$ at points $\omega_{l}(\mathbf{k})$. The self-energy part $\Sigma(\mathbf{k}, \omega)$ occurs through Green's function of a small polaron bounded to a spin waves. These peaks can be considered as the QP band of such complex states.

If we shall treat the width of the quasiparticle band $W$ as the difference $\omega_{l}\left(\mathbf{k}_{2}=(0,0)\right)-$ $\omega_{l}\left(\mathbf{k}_{1}=(\pi / 2, \pi / 2)\right)$ then $W$ turns out to be of order $J$ for small values of $J(J \simeq 0.1)$ in line with the results for the hole Green's function approach [12].

It is clear, that for small $J / \tau$ the concept of a small spin polaron fails and it is important to estimate the validity limits of this concept. Our calculations demonstrate, that the intensity of QP peaks and the structure $A_{p}(\mathbf{k}, \omega)$ do not change dramatically for $\mathbf{k}$, corresponding to the band bottom, up to $J / \tau=0.1$. For example, $Z_{p}\left(\frac{\pi}{2}, \frac{\pi}{2}\right) \approx Z_{p}(\pi, 0) \approx 0.50$ at $J / \tau=0.1$. So the $J / \tau$ lowest boundary value of the small spin polaron concept validity is lower than $J / \tau=0.1$

In Table 1 we give the numerical values $\omega_{l}(\mathbf{k})$ of the center position of the lowest $A_{p}\left(\mathbf{k}_{2}, \omega\right)$ peaks $\left(\omega_{l}(\mathbf{k})=\epsilon(\mathbf{k})\right.$ for $\mathbf{k}$ values where QP peak is observed) and their pole strength (area under the peak $) Z_{p}(\mathbf{k})$ for $\mathbf{k}=(\pi / 2, \pi / 2),(0,0),(0, \pi)$ and different values of $J$.

We do not represent the results for large $J, J \gg \tau$, as our approach in the present form fails to describe this limit. Here, from the very beginning we treat a small polaron by a single site operator $B_{\mathbf{r}}$ ( (4). For large $J$ the mean field static energy of such a state is proportional to $J$ and such a state is unstable. So in this limit we must extend the basis of site operators. The simplest way is to include in the basis the additional operator of a bare hole. In SCBA approximation this will lead to the system of two selfconsistent equations. As a result all effects of spin subsystem - hole interaction will be proportional to $\tau / J$. The more general procedure for the extending of the small polaron operator basis is outlined in [21. 


\section{SUMMARY}

We have studied the small spin polaron motion in the three-band model. The twotime retarded Green's function was calculated within the framework of self-consistent Born approximation for $32 \times 32$ cell lattice. We have shown that spin polaron of small radius represents a good approximation to the true quasiparticle low-energy excitation even at mean-field level. The account of the self-energy does not crucially change the polaron motion picture for realistic values of parameters. For quasimomenta $\mathbf{k}$ values, corresponding to the band bottom, most of the total spectral weight is concentrated in the quasiparticle peak (Table 【). In the same region of $\mathbf{k}$-space the shape of the QP dispersion curve $\epsilon(\mathbf{k})$ reproduces that of mean-field dispersion $\Omega_{\mathbf{k}}$ (Fig. 田).

We compare our results with previous investigations [11,12 that start from the bare hole. We see that the small polaron mean-field energy $\Omega_{\mathbf{k}}$ lies much lower than the QP pole

obtained from SCBA for the bare hole. Since $\Omega_{\mathbf{k}}$ determines the center of gravity for the Green's function spectral density the actual QP pole position (at least for the band bottom) should lie deeper in energy than $\Omega_{\mathbf{k}}$ (Figs. 3 and 6). This means that in the three-band model the important local correlations should be taken into account in zero approximation and small spin polaron should be constructed. Then the polaron scattering on spin waves is of less importance and it may be treated by perturbation methods.

The conclusion is that the low-energy physics of high- $T_{c}$ superconductors should be considered in terms of small spin polaron dynamics. In particular, that the problem of superconducting hole pairing must be treated as pairing of these quasiparticles rather than pairing of bare holes.

\section{ACKNOWLEDGMENTS}

We are grateful to O.A. Starykh and P.Horsch for valuable discussions and comments. This work was supported, in part, by the INTAS-PFBR organization under project No. 
INTAS-RFBR 95-0591, by the Russian Scientific Foundation for Fundamental Researches (Grant No. 95-02-04239-a), by Russian National program on Superconductivity (Grant No. 93080).

\section{APPENDIX. CHAIN REPRESENTATION AND INTEGRALS OVER THE SPECTRAL DENSITY}

The integration over spectral density could be done with the quadrature approach [27]. It is very efficient one when applied to the electron structure calculations [30]. Unfortunately, the spectral density we deal with has no upper bound and exponentially depends on the energy, i.e. it substantially differs from typical spectral density that appears in a bandstructure calculations. It turned out that the direct application of Nex's quadrature approach [27] is not stable numerically for our purposes. That is why for calculations of the integrals (27) we use the chain representation of continuous fraction. It means that the CF of the form

(21) may be interpreted as the Green's function $G(\omega)=\left\langle u_{0}\left|(\omega-\hat{h})^{-1}\right| u_{0}\right\rangle$ of the one particle tight-binding Hamiltonian $\hat{h}$ of the semi-infinite one-dimensional lattice, the $a_{n}, b_{n},\left|u_{n}\right\rangle$ being the site energies, nearest neighbor hoppings and on-site basis states respectively

$$
a_{n}=\left\langle u_{n}|\hat{h}| u_{n}\right\rangle, b_{n+1}=\left\langle u_{n}|\hat{h}| u_{n+1}\right\rangle .
$$

We introduce the eigenstates $\left|\psi_{m}\right\rangle$, and eigenenergies $E_{m}$ of the chain Hamiltonian

$$
\hat{h}=\sum_{m}\left|\psi_{m}\right\rangle E_{m}\left\langle\psi_{m}\right|
$$

then the Green's function spectral density becomes the local density of states on the zeroth site of the chain [26]

$$
A(\omega)=-\frac{1}{\pi} \operatorname{Im} G\left(\omega+\imath 0^{+}\right)=\sum_{m}\left\langle u_{0} \mid \psi_{m}\right\rangle \delta\left(\omega-E_{m}\right)\left\langle\psi_{m} \mid u_{0}\right\rangle .
$$

Then the following identities hold

$$
F=\int_{-\infty}^{+\infty} f(\omega) A(\omega) d \omega=\int_{-\infty}^{+\infty} f(\omega) \sum_{m}\left\langle u_{0} \mid \psi_{m}\right\rangle \delta\left(\omega-E_{m}\right)\left\langle\psi_{m} \mid u_{0}\right\rangle d \omega=
$$




$$
\sum_{m}\left\langle u_{0} \mid \psi_{m}\right\rangle f\left(E_{m}\right)\left\langle\psi_{m} \mid u_{0}\right\rangle=\left\langle u_{0}|f(\hat{h})| u_{0}\right\rangle .
$$

Nex has proved [27] that for a polynomial $f$ of the degree $2 n_{0}+1$ the integral $F$ for the infinite chain has the same value as the analogous integral for the truncated chain of the length $n_{0}+1$. The Hamiltonian of the truncated chain in the basis of states $\left\{\left|u_{0}\right\rangle \ldots\left|u_{n_{0}}\right\rangle\right\}$ has the form of the tridiagonal $\left(n_{0}+1\right) \times\left(n_{0}+1\right)$ matrix

$$
h_{T}=\left[\begin{array}{cccccc}
a_{0} & b_{1} & & & \\
b_{1} & a_{1} & b_{2} & & \\
& & \ldots & & \\
& & & & \\
& & & a_{n_{0}-1} & b_{n_{0}} \\
& & & & b_{n_{0}} & a_{n_{0}}
\end{array}\right] .
$$

Now, instead of integrating the spectral density function by $\omega$ we directly calculate the matrix function $f\left(h_{T}\right)$ in order to take $f_{00}$ matrix element. Then $F=f_{00}$, as it follows from the last identity of Eq. (39). We see that the answer for $F$ is expressed only through the first coefficients $\left\{a_{0}, \ldots, a_{n}, b_{0} \ldots, b_{n}\right\}$. 


\section{REFERENCES}

[1] E.Dagotto, Rev.Mod.Phys. 66, 763 (1994).

[2] S.Schmitt-Rink, C.M. Varma and A.E. Ruchenstein, Phys. Rev. Lett 60, 2793 (1988); F. Marsiglio et al.,Phys. Rev. B 43, 10882 (1991).

[3] C.L. Kane, P.A. Lee, and N. Read, Phys. Rev. B 39, 6880 (1989).

[4] G. Martinez and P. Horsch, Phys. Rev. B 44, 317 (1991).

[5] Z. Lui and E. Manousakis, Phys. Rev. B 44, 2414 (1991); ibid. 45, 2425 (1992).

[6] A.Sherman, M.Schriber, Phys. Rev. B. 48, 7492 (1993); ibid. 50, 12887 (1994); ibid. 50, 6431 (1994).

[7] N.M. Plakida, V.S. Oudovenko, V.Yu. Yushankhai, Phys.Rev. B 50, 6431 (1994).

[8] A. Ramsak and P. Prelovsek, Phys. Rev. B 42, 10415, (1990).

[9] V.J.Emery, Phys.Rev.Lett. 58, 2794, (1988).

[10] V.J. Emery and G. Reiter, Phys. Rev. B 38, 4547 (1988).

[11] O.F. de Alcantara Bonfim and G.F. Reiter, in Proceedings of the Univ. of Miami Workshop on Electronic Structure and Mechanisms for High Temperature Superconductivity, ed.J. Ashkenazi, New York, Plenum Press, 1991.

[12] V.V. Kabanov and A. Vagov, Phys. Rev. B 47, 12134 (1993).

[13] O.A. Starykh, O.F. de Alcantara Bonfim and G.F Reiter, Phys.Rev. B 52, 12534 (1995).

[14] F.C. Zhang and T.M. Rice, Phys. Rev. Lett B 37, 3759 (1988).

[15] A.F. Barabanov, L.A. Maksimov and G.V. Uimin, Pisma v Zh.Exp.Teor.Fis. 47, 532 (1988) [JETP Lett. 47, 622 (1988)]; Zh.Exp.Teor.Fis. 96, 655 (1989) [JETP 69, 371 (1989)] ; A.F. Barabanov, R.O.Kuzian and L.A. Maksimov, J.Phys. Cond. Matter 3, 9129 (1991). 
[16] J.G.Tobin, C.G.Olson, C.Gu et al, Phys.Rev.B 45, 5563 (1992).

[17] K.Gofron, J.C.Campuzano, H.Ding et al, J.Phys.Chem.Solids, 54, 1193 (1993).

[18] A.A.Abrikosov, J.C.Campuzano and K.Gofron, Physica C, 214, 73 (1993).

[19] D.S.Dessau, Z.-X.Shen, D.M.King et al, Phys.Rev.Lett 71, 2781 (1993).

[20] D.M.King, Z.-X.Shen, D.S.Dessau et al, Phys.Rev.Lett 73, 3298 (1994).

[21] A.F. Barabanov, V.M. Beresovsky, E.Žasinas, and L.A. Maksimov, Zh. Eksp. Teor. Fiz 110, 1480 (1996) [JETP 83, 819 (1996)].

[22] H.Shimahara and S. Takada, J.Phys.Soc.Japan 60, 2394 (1991).

[23] A.Barabanov, O.Starykh, J.Phys.Soc.Japan 61, 704 (1992); A.Barabanov, V.M.Berezovsky, Zh.Exp.Teor.Fis. 106, 1156 (1994) [JETP 79 (4), 627 (1994)].

[24] A.F. Barabanov, R.O.Kuzian and L.A. Maksimov Phys.Rev.B 55 , (1997) to be published.

[25] J.R. Schrieffer, J. Low. Temp. Phys. 99, 397 (1995).

[26] R. Haydock,in: Solid State Physics 35, eds. : H. Ehrenreich, F. Seitz and D. Turmbull, Academic, New York, 1980.

[27] C.M.M. Nex, J. Phys. A: Math. Gen. 11653 (1978).

[28] R. Haydock and C.M.M. Nex, J. Phys. C: Solid State Phys. 18 (1985) 2235.

[29] The Recursion Method and its Applications, eds. : D.G. Pettifor and D.L.Weaire, Springer, Berlin, 1985.

[30] R. Haydock and C.M.M. Nex, J. Phys. C: Solid State Phys. 17, 4783 (1984).

[31] Handbook of Mathematical Functions, eds. M.Abramowitz and I.A.Stegun, Nat. Bur. of Standarts, 1964. 
[32] A.P.Kampf and J.R.Schrieffer, Phys.Rev. B 42, 7967 (1990). 


\section{FIGURES}

FIG. 1. The coefficients $a_{n}$ and $b_{n}$ of the continued fraction expansion of $G_{p}(k, \omega)$ as functions on $n$ for $\mathbf{k}=(\pi / 2, \pi / 2)$ : a) $J=0.7 \tau, \mathrm{b}) J=0.1 \tau$. Calculated on the $32 \times 32$ cell lattice.

FIG. 2. Results for the hole Green's function $G_{h}(k, \omega)$ for the $t-J$ model calculated with the same parameters as in ref.( 四), $J=0.4 t, \mathbf{k}=(\pi / 2, \pi / 2), \eta=0.01,16 \times 16$ site lattice. a) The coefficients $a_{n}$ and $b_{n}$ of the continued fraction expansion of $G_{h}(k, \omega)$ as functions on $n$. b) Spectral function $A_{h}(k, \omega)$. c) Real part of the self-energy. d) Imaginary part of the self-energy. The unit of energy is $t=1$.

FIG. 3. Spin polaron spectral density $A_{p}(k, \omega)$, real and imaginary parts of the self-energy $\Sigma(k, \omega)$ calculated for $J=0.7 \tau$ and $32 \times 32$ cell lattice and different values of $k$. a) $k=(\pi / 2, \pi / 2)$, here we also reproduce the hole spectral function $A_{h}(k, \omega)$ obtained in Ref. [12] and pointed with h-arrows; b) $k=(0,0)$; c) $k=(\pi, \pi)$. In Figures (a-c) $\eta=0.002 \tau$, the sloping straight lines represent the function $\omega-\Omega(k)$. d) The dependence of the quasiparticle peak of $A_{p}(k=(\pi / 2, \pi / 2), \omega)$ for two values of the broadening factor $\eta$. The unit of energy is $\tau=1$.

FIG. 4. The dispersion of the QP band $\epsilon(k)$ and the mean field dispersion $\Omega_{k}$ along the symmetry lines in the Brillouin zone (see inset) for $J=0.7,32 \times 32$ cell lattice, $\eta=0.002$.

FIG. 5. $\quad A_{p}(k=(\pi / 2, \pi / 2), \omega)$ for $J=0.7$ and $\eta=0.002$ calculated for a) $N L=30$ and different lattice sizes; b) $32 \times 32$ cell lattice and different numbers of calculated CF levels $N L$.

FIG. 6. Spin polaron spectral density $A_{p}(k, \omega)$, real and imaginary parts of the self-energy $\Sigma(k, \omega)$ calculated for $J=0.1 \tau, \eta=0.002,32 \times 32$ cell lattice at three values of $k$ : a) $k=(\pi / 2, \pi / 2)$, b) $k=(0,0)$, c) $k=(\pi, \pi)$. 


\section{TABLES}

TABLE I. Position of the lowest by energy peak $\omega_{l}(\mathbf{k})$ and the area under the peak $Z_{p}(\mathbf{k})$ for different values of $J / \tau$ and $\mathbf{k}$

\begin{tabular}{ccccccc}
\hline \hline$J / \tau$ & $Z_{p}(0,0)$ & $\omega_{l}(0,0)$ & $Z_{p}(\pi / 2, \pi / 2)$ & $\omega_{l}(\pi / 2, \pi / 2)$ & $Z_{p}(\pi, 0)$ & $\omega_{l}(\pi, 0)$ \\
\hline 0.1 & 0.016 & -4.24 & 0.50 & -4.48 & 0.55 & -4.51 \\
0.3 & 0.039 & -3.37 & 0.72 & -4.09 & 0.714 & -4.13 \\
0.5 & 0.174 & -2.714 & 0.793 & -3.79 & 0.738 & -3.83 \\
0.7 & 0.347 & -2.25 & 0.823 & -3.52 & 0.808 & -3.56 \\
\hline \hline
\end{tabular}



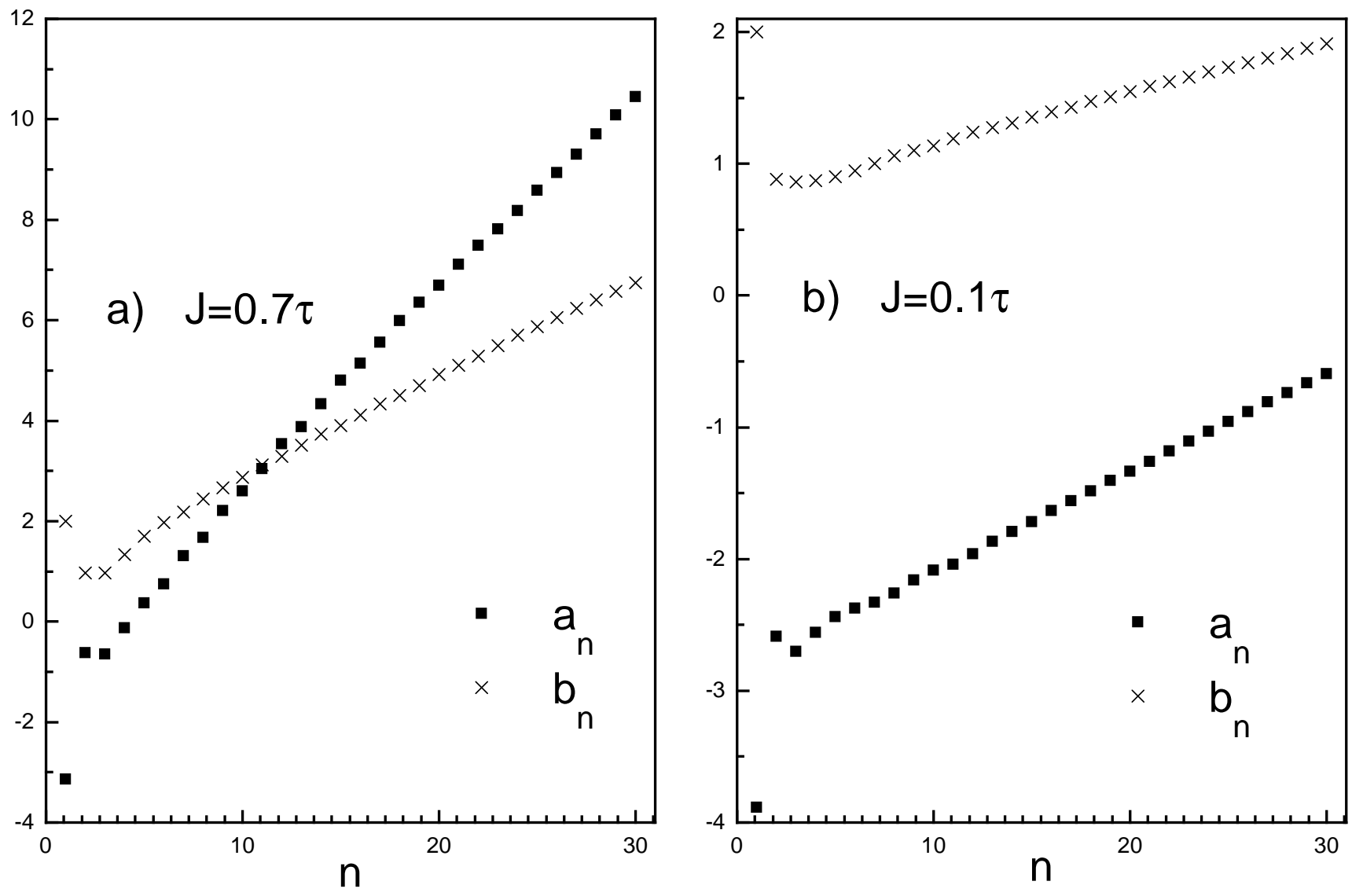

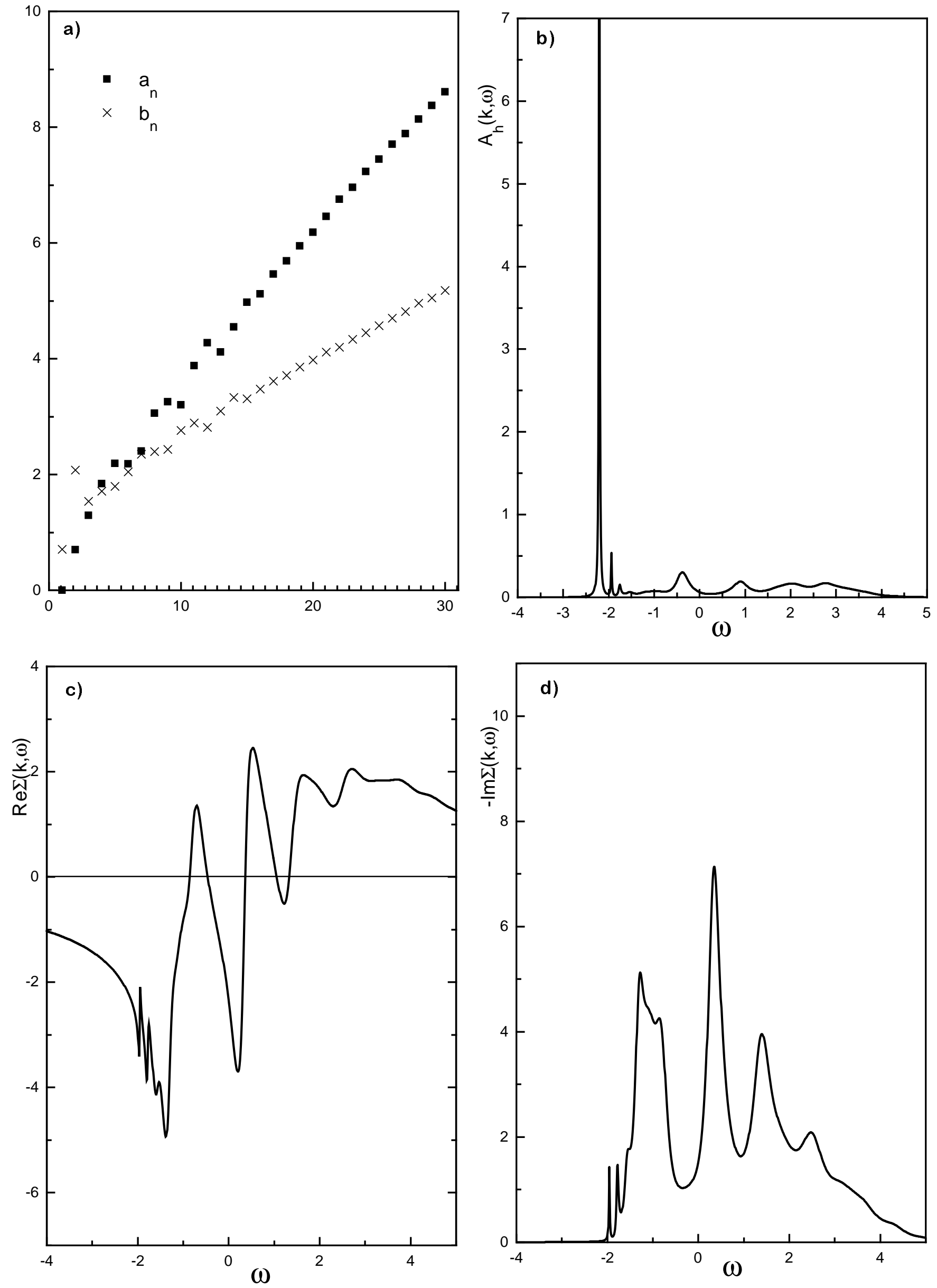

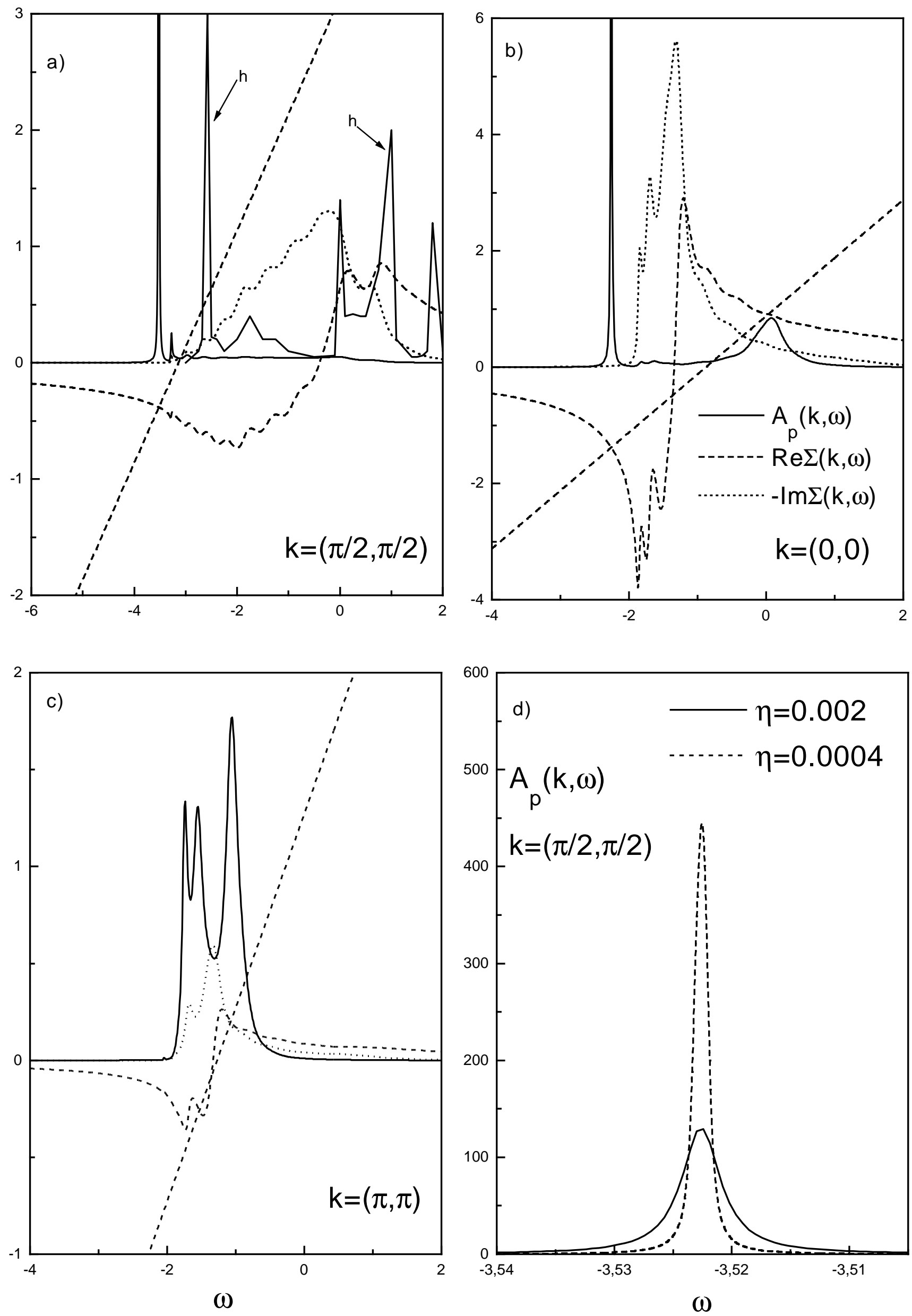


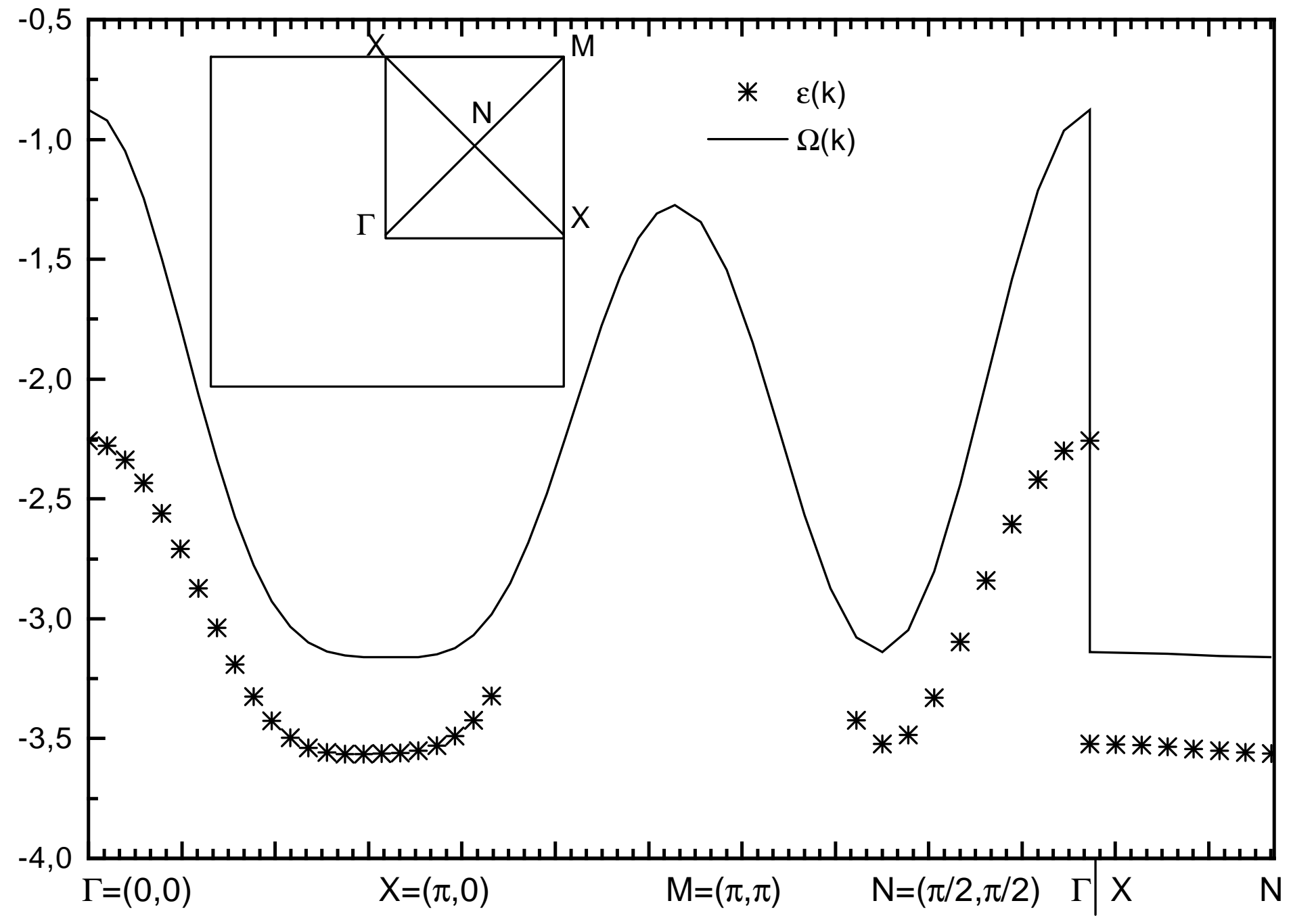



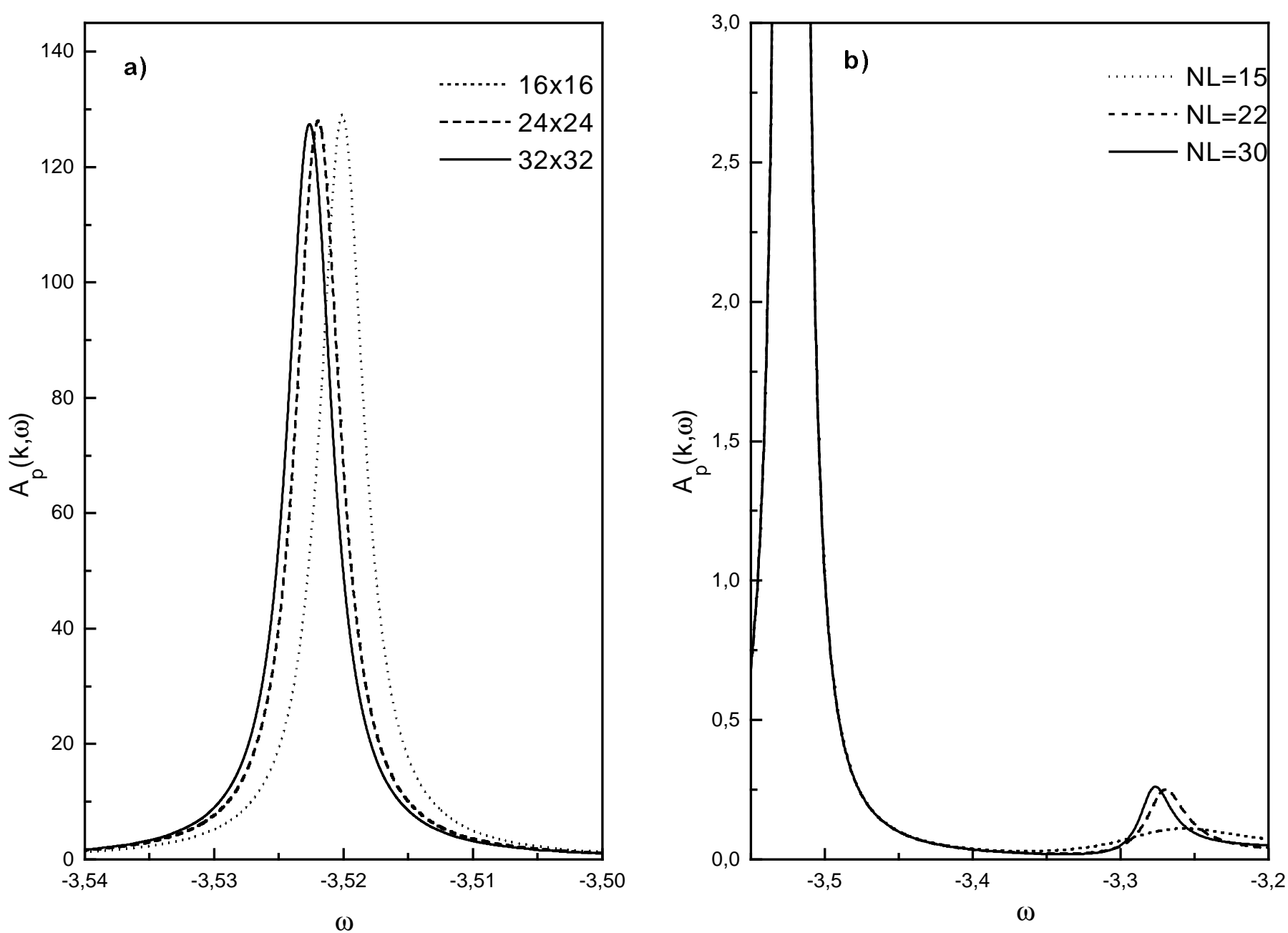


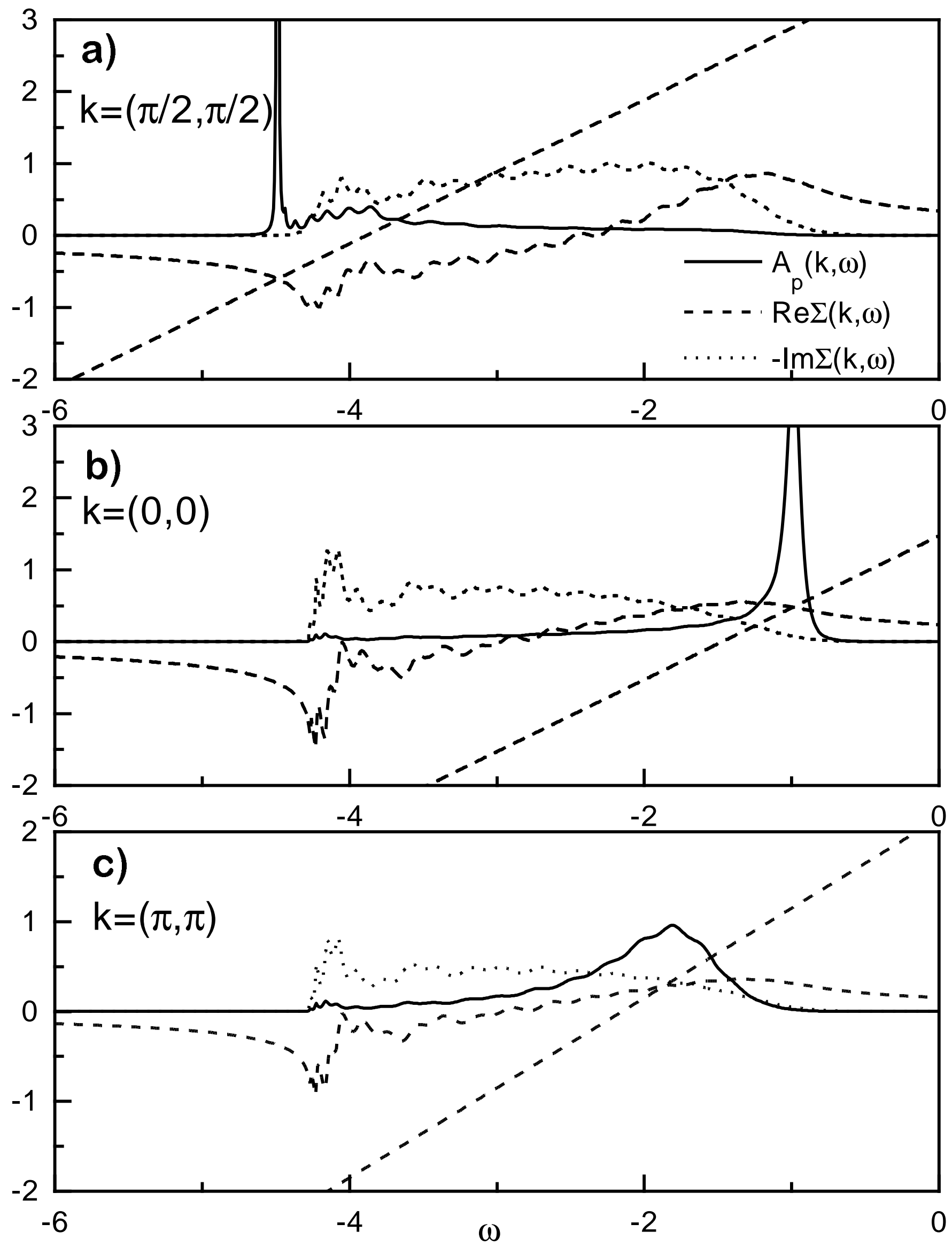

